\title{
The germline/soma dichotomy: implications for aging and degenerative disease
}

\begin{abstract}
Human somatic cells are mortal due in large part to telomere shortening associated with cell division. Limited proliferative capacity may, in turn, limit response to injury and may play an important role in the etiology of age-related pathology. Pluripotent stem cells cultured in vitro appear to maintain long telomere length through relatively high levels of telomerase activity. We propose that the induced reversal of cell aging by transcriptional reprogramming, or alternatively, human embryonic stem cells engineered to escape immune surveillance, are effective platforms for the industrialscale manufacture of young cells for the treatment of age-related pathologies. Such cell-based regenerative therapies will require newer manufacturing and delivery technologies to insure highly pure, identified and potent pluripotency-based therapeutic formulations.
\end{abstract}

First draft submitted: 10 October 2015; Accepted for publication: 2 March 2016; Published online: 24 March 2016

Keywords: age-related macular degeneration $\bullet$ aging $\bullet$ bone $\bullet$ brown adipose tissue - cartilage $\bullet$ choroid plexus $\bullet$ clonal embryonic progenitor cells $\bullet$ embryonic stem cells - endothelial progenitor cells $\bullet$ pluripotent stem cells

\section{The cellular basis of age-related pathology}

At a tissue level, human aging is often characterized by a progressive loss of normal histology. While the nature of this deterioration obviously varies by tissue type, the term 'morpholysis' (literally meaning 'dissolution of form') is generally applicable. Salient examples include osteoarthritis, agerelated macular degeneration, skin aging and osteoporosis. This morpholysis of aging differs from that associated with trauma or infection in that age-related morpholysis generally occurs in the absence of obvious external stimuli or immune infiltrates (i.e., osteoarthritis largely lacking the profound immunological component typical of rheumatoid arthritis). Instead, age-related morpholysis appears to reflect, at least in numerous cases, an intrinsic change in the particular tissue.
The assumption that aging is ultimately due to intrinsic changes in cells and tissues leads logically to the next question; namely, what are the molecular and cellular mechanisms behind the 'clockwork' that triggers the downstream pathological changes? Since the birth of cell biology in 19th century, a logical place to begin the search for possible clockwork mechanisms was within the cell itself. In charting the modern theory of heredity, the German naturalist August Weismann promulgated the simple proposition that the molecular basis of the immortal transmission of hereditary information from generation to generation is through direct transport of molecules through germline cells [1]. In contrast to the germline, Weismann proposed that the somatic cell lineages constituting the other tissues of the body are cast off each generation as a disposable appendage, therefore do not need the same replicative immortal-
Michael D West ${ }^{*, 1}$, Francois Binette $^{2}$, David Larocca ${ }^{3}$, Karen B Chapman ${ }^{3}$, Charles Irving ${ }^{4} \&$ Hal Sternberg ${ }^{1}$ 'BioTime, Inc., 1010 Atlantic Ave., Alameda, CA 94501, USA ${ }^{2}$ ReCyte Therapeutics, Alameda, CA 94502, USA

${ }^{3}$ OncoCyte Corporation, Alameda, CA 94502, USA

${ }^{4}$ Cell Cure Neurosciences, Jerusalem, Israel

*Author for correspondence:

Tel.: +1 5105213390 ext. 303

Fax: +1 5105213389

mwest@biotimemail.com
Future fsg 
ity. He therefore speculated that the first appearance of aging in evolutionary history coincided at about the time of the evolution of somatic cell types, and an associated divergence of the germline and soma replicative strategies. He surmised that "death takes place because a worn-out [somatic] tissue cannot for ever renew itself, and because a capacity for increase by means of cell-division is not everlasting, but finite" [2]. Weismann added that age-related disease could present itself before the complete exhaustion of cell proliferative capacity in a given tissue, "functional disturbances will appear as soon as the rate at which the worn-out cells are renewed becomes slow and insufficient" [2].

Weismann's prediction that somatic cells possessed an inherent finite replicative capacity was finally confirmed in the mid-20th century. Commonly designated the 'Hayflick limit' [3,4], this model system compares the molecular properties of cells early in their replicative lifespan with those at or near the end of that lifespan to search for alterations in, for example, gene expression, to find molecular pathways that may provide causal insights into age-related pathophysiology. In support of the physiological relevance of the cell senescence model of aging, the study of segmental premature aging syndromes such as Hutchinson-Gilford (progeria) and Werner Syndrome, each of which displays a premature onset of multiple age-related degenerative diseases such as atherosclerosis, osteoporosis, Type II diabetes, lipoatrophy and skin aging, also show a premature onset of in vitro cell senescence of cultured fibroblasts compared with normal control samples $[5,6]$.

Initial studies comparing young and senescent cells for alterations in functionality showed that while young cells were capable of dynamic alterations in the expression of secreted proteins such as proteases and protease inhibitors, senescent cells appeared to be arrested in a nonproliferative state, but paradoxically, one characterized by the secretome of activated young cells rather than quiescent ones. Since the normal maintenance mode of most stromal cells in vivo is quiescence, abnormally 'activated' senescent cells would be unable to proliferate in response to tissue damage, and potentially express secreted proteins that could lead to alterations in tissue maintenance. This inflammatorylike state of gene expression combined with cell cycle arrest is commonly designated senescence-associated secretory phenotype (SASP). Generally, activation markers are associated with growth factor or cytokineinduced proliferation and are downregulated with quiescence. The resulting profound fibroclastic nature of senescent cells displaying the SASP phenotype in what would otherwise be quiescence-inducing conditions may be due in part to constitutively high expression of interstitial collagenase and plasminogen activators $[7,8]$ that could have a dominant bystander effect on tissues. Perhaps only a minority of senescent cells in a tissue could shift the balance of synthesis to degradation of tissue extracellular matrix components leading to morpholysis (i.e. collagenolysis and elastolysis) seen in the intrinsic aging of skin [9] or other tissues.

But, what is the cause of the cell senescence in the first place? A theory for the primary mechanisms must take into account Weismann's suggestion, now considered empirical fact, that not all human cell lineages age. The germline lineage of cells has perpetuated life throughout the millennia, necessarily possessing replicative immortality $[10,11]$. This immortality is likely not dependent on meiosis. While most metazoans reproduce through unlimited cycles of sexual reproduction (alternating meiotic and mitotic events) that span the entire history of life on earth, some species, including occasional vertebrate species, can reproduce from unfertilized egg cells alone (parthenogenesis). Therefore, it is reasonable to postulate that sexual recombination or other events associated with meiosis may not be required for the immortality of the germline [12,13]. In addition, a valid model of cell aging needs to account for the potential of somatic cells to immortalize in the course of malignant transformation such as what occurs in the presence of viral oncogenes expressed by papillomaviruses, adenoviruses or Epstein-Barr virus. If cellular aging reflected very complex mechanisms or generalized entropy, how can cells immortalize at relatively high frequencies in the presence in some examples of a single viral oncogene?

A decade after Hayflick's report of human cell aging, Olovnikov proposed the hypothesis that the dichotomy of immortality/mortality in the germ-line/soma was due to an enzyme capable of maintaining the structure of chromosome ends (telomeres) in germline and cancer cells, while somatic cells lacked the activity and as a result experienced a progressive loss of telomeric DNA with each cell doubling eventually leading to senescence $[14,15]$. This telomere hypothesis of cellular aging and immortalization, is summarized in Figure 1. Telomere length is represented as terminal restriction fragment (TRF) length which is the average combined length of all chromosome telomeric repeats along with subtelomeric sequences between the restriction sites and the telomere end. Therefore, a TRF length of $5000 \mathrm{bp}$ may have 3000 bp of subtelomeric DNA and 2000 bp of telomeric repeats on average, but one or more telomeres that have become uncapped through a critical loss of repeats thereby triggering a p53-dependent DNA damage checkpoint cell cycle arrest.

TRF length is reported to be approximately $15 \mathrm{kbp}$ in human sperm cells, and to shorten in somatic cell lineages approximately $50-200 \mathrm{bp}$ with each round of 


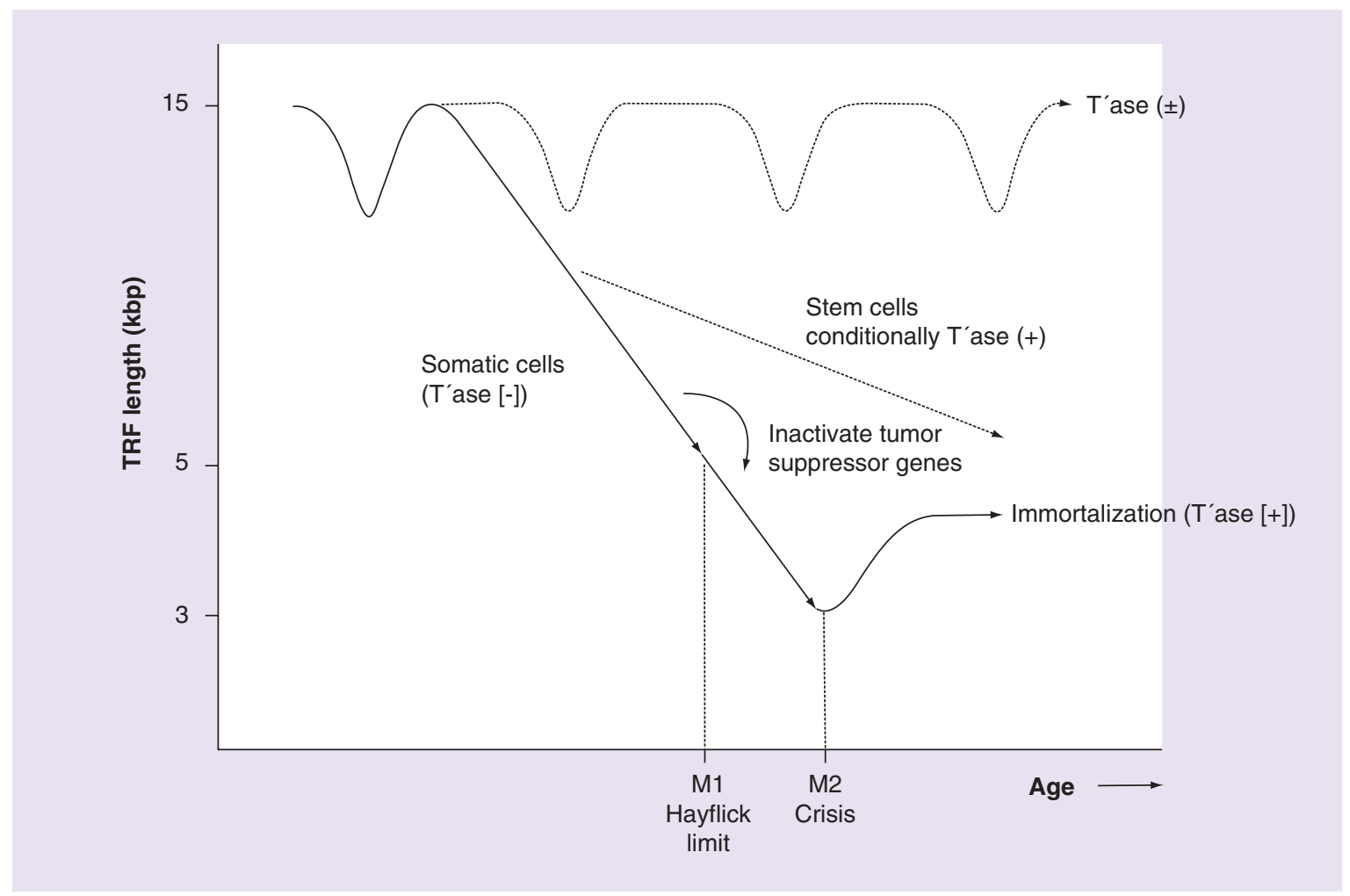

Figure 1. Telomere dynamics from embryogenesis to M1, M2 and immortalization. Somatic cells generally repress telomerase activity early in embryogenesis and as a result progressively lose telomere length following differentiation from the germline. By contrast, while perhaps oscillating during germ cell differentiation, the germline cell lineage maintains an immortal continuity from generation to generation. Finally, the abnormal reactivation of telomere maintenance during oncogenesis, typically after the inactivation of tumor suppression and extension of telomere loss to the point of multiple critically short telomeres, stabilizes telomere length in most malignant tumor types. This maintenance is most frequently through the reactivation of the telomerase catalytic component.

TRF: Terminal restriction fragment.

cell division until senescence occurs at about 5-7 kbp (Hayflick limit). Telomeric attrition in somatic cells lacking telomerase activity is therefore proposed to function as a 'replicometer', recording cell doublings as opposed to a 'chronometer', measuring metabolic time. The telomere theory received experimental support with studies demonstrating that the Hayflick phenomenon did indeed appear to reflect a mitotic rather than chronologic clock [16], and later that telomere shortening could be observed in somatic cell types compared with germline cells during human aging in vivo [17], even in stem cell compartments such as candidate hematopoietic stem cells [18]. Similar progressive shortening of telomeres was then observed in cultured human fibroblasts during passaging in vitro to senescence [19], while stabilization of telomeres was observed during the immortalization of cells by oncogenic viruses [20]. Furthermore, the replicative immortality of cancer cell lines correlated with the abnormal expression of telomerase activity (or more rarely, an alternative mechanism of telomere maintenance des- ignated 'ALT') and an ability to maintain telomere length over extended doublings [21,22]. Since these early observations, telomere dynamics have been the subject of extensive investigation. Based on studies of comparative zoology, the emerging evolutionary theory is that, at least for some taxa, the repression of telomerase activity combined with relatively short telomere lengths in the soma was selected for as it reduced the risk of malignancy in long-lived species. This model is therefore proposed to be a type of antagonistic pleiotropy [23].

The foregoing observations documented the correlation of telomere dynamics with that predicted by the Olovnikov hypothesis. The causal connection with senescence in vitro was demonstrated most convincingly by the exogenous expression of TERT in mortal differentiated cell types leading to their direct immortalization, apparently without transformation [24]. Additional data linking the biology to in vivo age-related degenerative disease resulted from the observation of increased incidence of damaged or 
shortened telomeres in cultured cells from patients with Werner syndrome [25] and progeria [26]. A common link between the two aging phenotypes having distinct mutated genes (WRN and LMNA) may be the mutual interaction of the encoded proteins of each gene with TRF2. The rescue from senescence in these progeroid cell types through the exogenous expression of TERT [27] provides further experimental support of the relevance of telomere shortening or damage to aging in vivo. Also providing support are experiments aimed at modifying telomere dynamics in mice. The breeding of Terc-knockout mice with Wrn mice leads to animals displaying human age-related pathologies not normally expressed in aged mice [28]. Also, transgenic mice overexpressing Tert together with tumor suppressor genes has been reported to extend normal lifespan [29]. In conclusion, growing experimental data support the hypothesis that the several degenerative diseases observed in common in normal aging and accelerated aging syndromes may also have common etiology in telomere dysfunction and may therefore logically be rescued by restoring youthful (long telomere) cell function in the target tissues afflicted with such pathology.

The delineation of some of the molecular pathways triggering cell senescence resulting from telomeric attrition or other genotoxic cascades such as those caused by oxidative stress on telomeres in telomerasenegative cells [30] (collectively referred to as senescence in this review), allowed for improved markers to determine if senescence occurs in vivo and if it correlates with age-related degenerative disease. Early observations showed that aged, as opposed to young tissue, shows a greater percentage of cells in the primary culture with a morphology like that of senescent cells [31]. This is difficult to reproduce however in comparing the replicative lifespan of cultures from individuals of differing ages, likely because of the overgrowth of the cultures by the youngest cells in the culture [32]. The use of markers of senescence such as lipofuscin [33] and secondary lysosomal enzymes [34], or more modern counterparts of lysosomal markers such as senescenceassociated $\beta$-galactosidase assay at $\mathrm{pH} 6(\mathrm{SA} \beta$-gal) [35], markers of cell cycle arrest such as those in the telomere damage-induced p53-p21-pRB pathway, or stress-induced p16-pRB pathway [36] or direct imaging of damaged telomeres such as senescence-associated DNA damage response foci (SDFs) or alternatively telomere dysfunction-induced foci (TIF) detected by the presence of $\gamma-H 2 A X$ (a histone $\mathrm{H} 2 \mathrm{~A}$ variant) [37] have proven useful in this regard. TIFs, for instance, have been reported to be useful markers for normal somatic cell senescence in vitro [38] as well as premature senescence in Werner syndrome [39] and progeria [40].
In summary, human somatic cell types typically display a mortal phenotype characterized by progressive telomere shortening when cultured in vitro and presumably during aging in vivo. Telomeres appear to be not merely a marker of cell aging, but may play a critical causative role, at least to some cell and tissue types. Nevertheless, many other cellular processes have been implicated in aging and cell aging in particular. These include alterations in such processes as autophagy, mitochondrial DNA mutations and other mitochondrial changes, free radical damage, deregulated nutrient sensing, epigenetic alterations, non-cell autonomous and endocrine/paracrine mediators and others [41]. Further research is required to clarify the order of cause and effect for each of these changes, some of which could be linked to telomere dynamics and some potentially to other developmental changes such as the embryonic-fetal transition where intrinsic tissue regeneration may be repressed. However, on the assumption that the germline lineage of cells escapes many of these age-related alterations with time, the culture of this entirely unique class of cells may provide a means of manufacturing young cells for the treatment of age-related degenerative disease.

\section{The pluripotent stem cell manufacturing platform}

In contrast to the mortal phenotype of cultured human somatic cells, human pluripotent stem (hPS) cells such as human embryonic stem (hES) cells or humaninduced pluripotent stem (hiPS) cells uniquely display replicative immortality when propagated in vitro. This appears to result from a relatively high level of telomerase activity [42]. Upon differentiation in vitro, $T E R T$ appears to be repressed relatively early with all continuously propagating differentiated or partially differentiated cell cultures lacking telomerase activity and showing progressive telomere shortening and a finite replicative lifespan when cultured in vitro [43]. This natural replicative immortality of most pluripotent stem cells appears to reflect less the ability of these cells to normally self-renew (a defining characteristic of a stem cell) than their nearness to the branch point of germline and somatic cells on the ontological tree. Indeed, the concept of the self-renewal of stem cells being equated with replicative immortality, at least in the case of the human species, is probably misplaced. While mouse stem cells and numerous other mouse cell types spontaneously immortalize at a relatively high frequency, human cell types typically do not, and there has yet to be a functional human stem cell type observed to maintain telomere length and display replicative immortality in vitro. While hES and hiPS cells are clearly an exception, it is important to note 
that they are not normally stem cells in vivo. That is, there is no niche of pluripotent stem cells in the normal human body that have the potential to self-renew or differentiate, unless one considers the ephemeral appearance of the inner cell mass and epiblast of the human blastocyst a 'niche'. Therefore, human pluripotent stem cells appear to differ from all reported human fetal and adult-derived stem cells in regard to their simultaneous potential for replicative immortality and pluripotency. There have been numerous reports of the initial characterization of immortal and pluripotent cells from human fetal or adult sources $[44,45]$, but to date, there is scant or contradictory confirmation in the literature [46].

\section{Rescue from developmental aging}

Cells clinically useful for transplantation would require a cost-effective method of solving the problem of histoincompatibility. The demonstration that somatic cell nuclear transfer (SCNT) is capable of reprogramming mammalian somatic cells to pluripotency, indeed, pregnancies and live births [47], led to the logical question of its use to 'reverse the developmental aging' of aged human somatic cells and the generation of patient-specific young cells of all types for use in regenerative medicine [48]. The 'age-reversal' of somatic cells reprogrammed by SCNT or iPS cell technologies can be observed through the resetting telomere length, reversal of progerin accumulation in reprogrammed cells from progeria patients, restoring cells to an embryonic state and even live births in nonhuman species as well as by other markers of aging as described in this review. However, while it is the thesis of this review that this 'age-reversal' as applied to reprogrammed human somatic cells is valid, the rigorous demonstration of the fact would require human experimentation difficult or unethical to undertake. Therefore, the authors use the term 'the reversal of the developmental aging' of cells to refer to only that which has been documented to date in the literature, in other words, the restoration to pluripotency and ES cell telomere length to aged somatic cells.

Initially, in regard to Dolly, the first mammal cloned from a somatic (mammary epithelial) cell, reports suggested that the animal was born with abnormally short (presumably not reset) telomere length [49]. However, numerous subsequent studies in bovine and other species have shown telomere extension and extended somatic cell replicative lifespans to even longer lengths than that observed normally [50]. Similar results have been observed in other laboratories though differences may be linked to the choice of the somatic cell source or other unidentified parameters in the protocols used [51]. Further experimentation will need to be per- formed to determine whether the use of epithelial cells as opposed to connective tissue fibroblasts as somatic cell sources truly influences the reprogramming of telomere length.

SCNT is not only technically challenging, its widespread use would require a large supply of human oocytes. Therefore, the simplification of the reprogramming protocol to only the exogenous administration of defined transcriptional regulators to produce iPS cells has streamlined the process in a manner that makes it practical to implement in most laboratories [52]. While telomere length extension back to embryonic length is not a universal property of aged somatic cells reprogrammed using all iPS cell protocols, it nevertheless can be achieved [43]. As in the case of the exogenous expression of TERT, transcriptional reprogramming appears to also rescue Werner syndrome and progeria fibroblasts from senescence [53,54], further supporting the potential utility of the technology in reversing age-related pathology in the diseases held in common between normal aging and these segmental progeroid disorders.

Since the immortality of pluripotent stem cells enables the indefinite expansion of master cell banks and subsequently differentiating working cell banks into scalable young and diverse somatic cell types, it may also allow for complex targeted homogeneous genetic modifications similar to that used in the production of transgenic mice. A commonly overlooked advantage of immortal master cell banks is that they allow cells that have been successfully targeted with a genomic sequence modification to be clonally expanded into new master cell banks that can subsequently be modified again, indeed endlessly, yielding highly sophisticated modifications with homogeneity. Any genetic drift or unintended alterations can then easily be identified using current genomic sequencing technologies [55]. These modifications can include ones intended to induce allogeneic immunotolerance tolerance of a graft such as the overexpression of PD-L1 and CTLA4-Ig [56], or the elimination of HLA class I genes [57] that provides the additional benefit of potentially allowing an off-the-shelf product simplifying regulatory approval. Therefore, the unique property of hPS cells to propagate robustly and indefinitely facilitates a strategy of producing cGMP-grade therapeutically useful master cell banks that allow the continuous and unlimited manufacture of product of a uniform genotype.

\section{The differentiation of hPS cells into therapeutic products}

In addition to the benefit of the immortal proliferation of undifferentiated hPS cells, the cells also have 
the advantage of their potential to cascade into all the 1000-plus somatic cell types in the human body including embryonic progenitors (i.e. cells displaying a phenotype characteristic of that prior to the embryonicfetal transition). Cells and tissues from the developing mammal in the period of embryonic development, but before fetal development, show a remarkable potential for tissue regeneration including scarless wound repair such as well studied in the skin [58]. Some multicellular organisms, including some vertebrates, show a profound ability to regenerate complex tissues such as limbs after amputation. Vertebrates that display such a profound regenerative capacity generally do not show age-dependent loss of the regenerative potential [59]. Therefore, it seems likely that gene expression patterns of the developing embryo are maintained through some undefined process of heterochrony in these animals compared with humans. The ability to manufacture primitive human embryonic progenitor cell types could potentially allow easy access to candidate cell populations with this phenotype.

However, the vast pluripotency of hPS cells is as much a challenge as it is opportunity. Strict quality control standards enforced by the US FDA for cells used in human therapeutics demand a high degree of reproducibility in manufacturing identified and purified cell types [60]. Since the fate space of hPS cells exceeds 1000 distinguishable cell types, demonstrating purity and identity will likely be challenging [61] .

A simple solution to this challenge is to scale product from single cells in transit from pluripotency to fully differentiated cells. These cultures also known as clonal embryonic progenitor cell lines, have been reported to be expandable in greater than 200-fold diversity [62], with numerous lines being multipotent, capable of making, for instance, site-specific osteochondral cell types [63], meningeal and choroid plexus cells [64] as well as other therapeutically useful cell types. The relatively long initial telomere length of the parental hPS cell master cell banks gives clonal lines, while mortal, a correspondingly long replicative lifespan facilitating industrial scale-up [65].

\section{Injectable biocompatible matrices}

When most anchorage-dependent somatic cell types are formulated in saline and injected into solid tissue, they typically undergo massive anoikis (apoptosis) [66]. Therefore, a prerequisite for the aforementioned cellbased therapies directed at solid tissues are formulations of cells combined with critical extracellular matrix components such as hyaluronic acid and type I collagen. Since cell matrices also require a degree of rigidity to prevent anoikis, it is necessary for matrix components to be injected safely in the presence of cells and then for covalent cross-linking of the matrix components to occur to a defined degree of rigidity.

Thiol-modified hyaluronan and gelatin hydrogels can be produced using polyethylene diacrylate crosslinkers. When the components are mixed in the presence of cells, a viscoelastic hydrogel forms in approximately $20 \mathrm{~min}$ at room temperature where the compliance (stiffness) of the hydrogel can be varied from 25 to 3500 Pa by altering the amount of crosslinker. Such combination cell-matrix formulations appear to increase cell survival for cells transplanted into such diverse tissues as the liver, brain, heart as well as other tissues [67]. An alternative strategy is the assembly of cells onto decellularized tissues. The latter strategy has the advantage of a more robust organ-shaped construct, but generally has the challenge of integrating cells into a tight 3D matrix construct, and therefore of sufficient cellularity to function adequately in vivo.

\section{Potential therapeutic applications in age-related degenerative disease}

Assuming that previously-mentioned vertebrates with a profound capacity for tissue regeneration do so because they are either transiently in a developmental stage prior to the embryonic-fetal transition or are displaying a heterochronous retention of embryonic patterns of gene expression in adulthood, the question arises whether hPS cell-derived clonal embryonic progenitor cell lines with long initial telomere length also displaying a pre-fetal pattern of gene expression could prove useful in regenerating tissues afflicted with age-related degenerative disease. We will briefly discuss some salient examples of where such therapies may prove interventional.

\section{Skin aging}

Dermal fibroblasts are one of the most common cell types utilized in cell aging research [9]. Evidence of up to $15 \%$ of dermal fibroblasts displaying senescence in aging primates has been reported [68]. The morpholysis in aging skin can be profound, with a marked loss and fragmentation of collagen fibers and elastolysis. The study of the secretome of aging dermal fibroblasts showed that while young cells are capable of becoming activated in the presence of serum growth factors and entering the cell cycle while up-regulating metalloproteinases such as interstitial collagenase (MMP1) [7] or upon removal of such stimulation returning to quiescence, exiting the cell cycle and down-regulating MMP1, senescent cells differ in that they are blocked in a terminally activated, yet nonproliferating state [8]. Besides contributing to the aged appearance to the skin, dermal fibroblast senescence may contribute to impaired wound healing and chronic nonhealing ulcers 
in the aged. The production of young fibroblasts may facilitate wound healing in the elderly. Consistent with this is the observation that adenoviral-based exogenous expression of TERT in a rabbit model of skin aging led to markedly improved wound repair [69]. In addition to proliferation-competent cells, wound healing could be facilitated by the induction of scarless wound repair such as that observed in the embryonic phases of development [70]. The isolation of such cells from pluripotent stem cells and their use in stimulating rapid and scarless wound repair in the aged could greatly reduce the costs of managing chronic wounds in these patients [58].

\section{Musculoskeletal degeneration}

Osteoarthritis is an age-related degenerative disease. The pain associated with the disorder is a leading complaint in the elderly and the most common disease of the joint [71]. While the etiology appears to be multifactorial, aging is a leading risk factor [72]. Articular chondrocytes are generally considered to be essentially postmitotic throughout adulthood, nevertheless, at sites of cartilage injury, clonal proliferation of chondrocytes has been observed, potentially leading to senescence. Alternatively, stresses on articular chondrocytes from sarcopenia or damage to the meniscus may lead to fragmentation of telomeres. The resulting senescent cells, like dermal fibroblasts, may overexpression metalloproteinases compared with metalloproteinase inhibitors, and the result is a cascade of disease, proliferation and more disease.

During the aging process, degeneration of the intervertebral disc can lead to considerable pain and disability as well, including low back pain. Like other connective tissues, cell senescence markers such as $S A \beta$-gal, DNA damage checkpoint markers, metalloproteinases and shortened telomere length are observed [73] that mimick cell senescence changes in nucleus pulposis cells aged in vitro [74]. In the case of the loss of articular cartilage such as commonly occurs in osteoarthritis, or the loss of structure to the intervertebral discs, patients could potentially benefit from the transplantation of hPS cell-derived progenitors to those respective tissues. The use of hPS cell-derived clonal embryonic progenitor cell lines has been shown to lead to scalable progenitors of diverse site-specific skeletal tissues that have potential to regenerate structure in animal models [75]. Further studies will be required to determine the utility of these novel and diverse embryonic cell types in contrast to the more commonly studied adult-derived mesenchymal stem cells.

\section{Age-related macular degeneration}

Age-related macular degeneration (AMD) remains a leading cause of blindness in many countries around the world, afflicting over 30 million people [76]. The wet form of the disease can be effectively prevented with regular administration of angiogenesis inhibitors such as ranibizumab or aflibercept, however, this form of the disease accounts for only about $10 \%$ of disease prevalence in the USA. Some $90 \%$ of patients have the dry form represented by slow progression of geographic atrophy which can also cause blindness and for which there is currently not approved therapy in the USA. The etiology of this age-related degenerative disease is thought to be the age-related loss or dysfunction of retinal pigment epithelial (RPE) cells [77,78] that normally provide diverse types of support for the neural retina and produce a natural angiogenesis inhibitor (PEDF) [79], potentially reflecting the senescence of the cells [80]. Consistent with this is the observation of a loss of PEDF (also known as EPC-1) expression in cells grown to senescence in vitro [81]. The derepression of angiogenesis inhibition in the aging retina may therefore lead to increased neovascularization and the wet form of the disease. The loss of RPE cells, or reduced phagocytosis or autophagy may account in part for the dry form of the disease as well [82].

Linking the loss of healthy RPE cells to this devastating disease led to the proposal that RPE cell grafts may protect against progression of one or both forms of the disease. Initial attempts sourced cells from donated eyes, autologous peripheral retina or from cells scaled in vitro. While some efficacy was reported, the limited scalability of RPE due to de-differentiation and senescence led to a search for an alternative source [83].

Pluripotent stem cells have been reported to be a robust source of young RPE cells [84,85]. Animal preclinical studies demonstrate potential safety and efficacy in the Royal College of Surgeons (RCS; London, UK) rat model retinal degeneration [86]. At least three clinical studies using hES-derived RPE cells are underway, one utilizing iPS cell-derived grafts currently on hold for quality control issues related to drift in genotype [87]. Since hPS cell-derived RPE cells appear to have little immunogenicity and perhaps even to be immunosuppressive [88], unmodified cells may be tolerated long-term with only transient immunosuppression. Human clinical trials utilizing doses expected to be potentially therapeutic should soon yield useful data to evaluate this application of hPS cell-derived grafts for an age-related degenerative disease.

\section{Age-related neurodegenerative disorders}

The aging of the CNS is correlated with several degenerative diseases such as Parkinson's disease (PD), Alzheimer's disease (AD), and stroke [89]. The focal and profound loss of dopaminergic neurons in the midbrain in PD, and evidence of potential efficacy of 
transplanted fetal-derived neurons, has led to a search for hPS-derived cells potentially useful in treating PD [90]. Animal nonclinical data support the potential safety and utility of such cells [91]. Human clinical trials of parthenogenetic pluripotent stem cell-derived dopaminergic neurons for the treatment of $\mathrm{PD}$ are currently underway with initial results expected within the decade. By contrast to $\mathrm{PD}$, the pathology of $\mathrm{AD}$ is diffusely disseminated. However, localized degeneration of the choroid plexus has been implicated in the pathogenesis of the disorder. The transplantation of progenitors to the choroid plexus to increase the turnover rate of cerebral spinal fluid may therefore provide a novel therapeutic strategy for AD. In the case of stroke, nonclinical studies of the utility of embryonic neuroepithelium in regenerating damaged neocortex, as well as the use of hPS cell-derived cells to express neurotrophic factors such as BDNF are currently underway.

\section{Age-related metabolic disorders}

Aging is often associated with profound alterations in metabolism such as generalized loss of subcutaneous fat, central visceral obesity, Type II diabetes, hypertension and atherosclerosis. It has been proposed that the age-dependent loss of white subcutaneous fat leads to decreased clearance of triglycerides and increased deposition in ectopic sites including coronary arteries [92]. Progeria shows an almost a complete loss of peripheral subcutaneous adipose tissue also associated with Type II diabetes and coronary disease [93] and similar alterations are seen in Werner syndrome [94].

In addition to the age-dependent loss of subcutaneous fat, there is a profound loss of brown adipose tissue with age [95]. It appears likely that these agedependent redistributions of adipose tissue play an important role in aspects of the metabolic syndrome in aging. The transplantation of subcutaneous fat or brown adipocytes may provide a novel modality to inducing weight loss and increasing triglyceride and glucose clearance [96]. Pluripotent stem cells may provide a useful source of these cells if they can be reliably manufactured in a highly purified and identified state. The transplantation of the cells in HyStem hydrogels may promote survival of these grafts and promote differentiation [97].

\section{The hematopoietic system}

The ease of access of blood cells for TRF length analysis has led to a plethora of studies of telomere length as a function of age, age-related diseases [98] and even lifestyle practices [99]. Many of these studies do not, however, control for complex changes in composition of circulating peripheral blood lymphocytes (PBLs) in response to such environmental factors such as expo- sure to pathogens, or endocrine factors. In addition, many early studies have used relatively crude measures of mean TRF length on a Southern blot that is complicated by individual variations in the subtelomeric X region of the fragments and/or incomplete enzymatic digestion. Newer assay methods such as qPCR-based assays, quantitative FISH (Q-FISH) in interphase cells [100] or the measurement of individual telomeres [101] when implemented on a large scale may provide more precise and reproducible results. Nevertheless, it seems clear that telomere length decreases with age in PBLs, reaching a length in centenarians comparable to that observed in fibroblasts grown to the Hayflick limit in vitro [102].

While the pathological impact of senescence in blood cells is still largely unknown, the correlation of short telomeres in CD8 ${ }^{+}$lymphocytes in AIDS [103], suggests that senescence may explain the similarities of AIDS and age-related immunodeficiencies [104]. In addition, it is common knowledge that aged individuals make relatively poor bone marrow stem cell donors [105]. Therefore, whether to provide young proliferationcompetent blood cells for adoptive immunotherapy or for reconstituting the entire hematopoietic system, hPS cell-derived grafts may benefit aged patients.

In addition, it has been suggested that circulating endothelial progenitor cells (EPCs) originate from bone marrow stem cells. These cells are believed to circulate and repair injured endothelium throughout the body [106]. Since circulating EPC telomere length has been observed to decrease with age [107] and endothelial dysfunction has been implicated in a number of age-related changes in the vascular system including those associated with the highest risk of mortality in the USA [108], hPS cell-derived EPCs may also be a useful regenerative strategy. Old cows transplanted with nuclear transfer-derived fetal liver CD $34^{+}$fetal hematopoietic stem cells showed engraftment even without ablation, and apparent incorporation of derivative cells into vascular endothelium [109].

\section{Conclusion}

The rapid increase in the number of elderly people around the world places an ever-increasing strain on the healthcare infrastructure of many industrialized countries. The greatest expense is related to current treatment modalities for chronic disease. In the USA alone, chronic disease accounts for some $80 \%$ of the US $\$ 3$ trillion expense annually [112]. Since most current therapeutic regimens for chronic age-related diseases are largely palliative, they represent a major source of the economic burden [110]. This demographic trend underscores the growing need to understand aging on a cellular and molecular level and to intro- 
duce novel cost-effective therapies. Understanding the unifying cellular and molecular pathways underlying these pathologies could lead to innovative strategies that could both alleviate human suffering and reduce healthcare expenditures by collapsing the time of morbidity, reducing frailty and increasing the independence of the aged.

It is possible that such unifying pathways can be identified by examining the molecular mechanisms aging holds in common with segmental progeroid syndromes. In particular, one intriguing avenue of study is where such age-related diseases as osteoporosis, skin aging, coronary disease, cataracts, Type II diabetes and lipoatrophy (diseases held in common between progeroid syndromes and normal aging) are linked to genes that encode proteins with an apparent function in maintaining telomeres and hence related to cell replicative lifespan. However, the gerontological community has yet to come to a consensus on these mechanisms [111].

Pluripotent stem cells appear to be naturally immortal progenitors to all somatic cell lineages. To meet the needs of many millions of individuals with chronic degenerative conditions, we propose that master cell banks of pluripotent cells genetically modified to escape immune surveillance and make off-the-shelf universally transplantable allogeneic cells is likely the most feasible strategy to manufacture cost-effective product. These master cell banks can then be utilized to produce clonal embryonic progenitors to simplify control of the quality (identity and purity) and the use of biocompatible matrices to increase survival of the cells and reduce undesired migration from the graft site may increase the efficacy and safety of the therapies.

In this review, we propose that hPS cells derived from either preimplantation embryos or produced from somatic cells induced into pluripotency (assuming the latter have reset telomere length back to germline length) are comparable to those providing a lifetime of functionality to the tissues in the developed human. If products manufactured from these cells retain the regenerative potential observed in the embryonic phases of development, they may have even more benefit to the patient than fetal or adult-derived cells. Ultimately, the safety and efficacy of any one clinical applications of the foregoing will depend on the complexities of individual pathology, genetic background and the unknown variable of heterochronic transplantation. Ongoing clinical trials of hES and hiPS cell-derived therapeutics will likely yield data useful in evaluating the safety and efficacy in defined disease states. However, many questions will remain to be answered. Important questions relate to the safety of cellular grafts in a heterochronic setting. That is, how do young or even embryonic progenitors to a particu- lar tissue compare to adult or fetal-derived cell grafts? Another important safety question will be how safe are cell grafts containing undefined cellular contaminants that while not pluripotent, may nevertheless be multipotent embryonic progenitors. What are those progenitors, do they survive in ectopic sites, and do they continue to differentiate to what would have been their normal differentiated state?

\section{Future perspective}

The rising number of elderly in the industrialized countries such as the USA and the associated escalation in the incidence of age-related degenerative diseases challenges the resources of our current healthcare system. Therefore, there exists a significant and growing need for understanding the molecular and cellular underpinnings of aging and providing novel modalities for the costeffective treatment of its sequelae. An emerging therapeutic strategy harnesses the natural replicative immortality of germline cells as it occurs in pluripotent stem cells, as a renewable source of transplantable young cells that can be used to restore healthy function to aging tissues. If modified to generate allogeneic off-the-shelf products, such technology has the potential to greatly reduce the human and economic costs of chronic degenerative disease. Such therapies will require methods for the reproducible manufacture of highly defined and purified cell types as well as biocompatible matrices to promote reliable regeneration of normal $3 \mathrm{D}$ tissue in vivo.

\section{Financial \& competing interests disclosure}

This work was funded by BioTime, Inc., OrthoCyte Corporation, ReCyte Therapeutics and OncoCyte Corporation (Alameda, CA, USA), and Cell Cure Neurosciences, Jerusalem, Israel. F Binette, H Sternberg and MD West are employees of BioTime, Inc. (Alameda, CA, USA). D Larocca is an employee of ReCyte Therapeutics (Alameda, CA, USA). KB Chapman is an employee of OncoCyte Corporation (Alameda, CA, USA). C Irving is an employee of Cell Cure Neurosciences (Jerusalem, Israel). F Binette is also an employee of OrthoCyte Corporation (Alameda, CA, USA). ReCyte Therapeutics, OncoCyte Corporation, Cell Cure Neurosciences and OrthoCyte Corporation are subsidiaries of BioTime Inc. The authors have no other relevant affiliations or financial involvement with any organization or entity with a financial interest in or financial conflict with the subject matter or materials discussed in the manuscript apart from those disclosed.

No writing assistance was utilized in the production of this manuscript.

\section{Open access}

This work is licensed under the Attribution-NonCommercialNoDerivatives 4.0 Unported License. To view a copy of this license, visit http://creativecommons.org/licenses/by-nc-nd/4.0/ 
Executive summary

The cellular basis of age-related pathology

- The advent of cell biology in the 19th century led to the detailed description of the cellular composition of tissues in the course of aging and the question of whether aging is caused by changes intrinsic or extrinsic to cells.

- August Weismann proposed that somatic cells, as opposed to germline cells have an intrinsic clocking mechanism that limits their replicative capacity while the germline maintains the immortal propagation of individuals.

- The finite replicative capacity of human somatic cells was first demonstrated by Hayflick in the 1960 s and cells from premature aging disorders such as progeria and Werner syndrome were shown to have a shortened cell lifespan.

- The senescence of cells may lead to pathology either directly through the loss of cells or from associated alterations in gene expression such as the senescence-associated secretory phenotype.

- The telomere hypothesis first proposed by Olovinokov now has significant support as a fundamental cause of the dichotomy of mortality and immortality in somatic versus germline cells, respectively.

The pluripotent stem cell manufacturing platform

- Pluripotent stem cells express relatively high levels of TERT and telomerase activity and generally maintain telomeres at a length comparable to that of sperm, but repress TERT expression upon differentiation into somatic cell types.

Rescue from developmental aging

- Somatic cell nuclear transfer techniques, like normal reproduction, are capable of resetting telomere length and cell lifespan to aged somatic cells.

- The reprogramming of aged somatic cells by the administration of exogenous transcriptional regulators can reverse the developmental aging of the cells (i.e., reset telomere length as well as differentiation to pluripotency).

- The use of immortal and pluripotent master cell banks allows endless rounds of precise genetic modification followed by clonal expansion, with subsequent differentiation into diverse therapeutically useful cell types.

The differentiation of human pluripotent stem cells into therapeutic products

- The use of pluripotent stem cells to derive therapeutic cell formulations is complicated by the vast diversity and relative lack of precise markers of the thousands of derivative cell types. The long telomere length of pluripotent stem cells allows the clonal expansion of primitive embryonic progenitors simplifying manufacturing protocols.

Injectable biocompatible matrices

- Chemically modified hyaluronic acid and collagen-based matrices allow in vivo covalent cross-linking of cells in 3D constructs, thereby increasing postengraftment survival while decreasing migration to undesired ectopic sites.

Potential therapeutic applications in age-related degenerative disease

- Skin aged in vivo shows markers consistent with a role of cell senescence in the pathological changes observed. The identification of clonal embryonic progenitors that display an embryonic, as opposed to fetal/adult phenotype may promote a scarless regenerative potential.

- Senescent markers in the weight-bearing joints of patients with osteoarthritis or intervertebral joint degeneration may benefit from embryonic progenitors to those tissues capable of engrafting and regenerating healthy histology.

- Age-related macular degeneration is characterized by a loss and/or dysfunction of the retinal pigment epithelium. The replacement of such cells which play an important role in inhibiting angiogenesis as well as supporting the function of the neural retina may therefore provide an important therapeutic strategy to slow the progression of the dry form of the disease.

- Age-related neurodegenerative disorders such as Parkinson's disease may benefit from the transplantation of midbrain dopaminergic neurons. Clinical trials using human pluripotent stem cell-derived formulations are expected to begin trials within the decade.

- Metabolic disorders such as Type II diabetes and central obesity may benefit from the transplantation of brown adipocytes. The formulation of such cells in an injectable matrix such as that comprised of crosslinkable hyaluronic acid and collagen may provide the means of establishing a therapeutic dose of these cells to lower circulating glucose levels and cause weight loss.

- Nucleated blood cells are well documented for age-related telomeric attrition. In addition, protocols are well established for the transplantation of hematopoietic stem cells. The transplantation of histocompatible young hematopoietic stem cells may benefit many patients currently needing such therapy. 


\section{References}

1 Kirkwood TB, Cremer T. Cytogerontology since 1881: a reappraisal of August Weismann and a review of modern progress. Hum. Genet. 60(2), 101-121 (1982).

2 Poulton EB, Schönland S, Shipley AE. Essays Upon Heredity and Kindred Biological Problems (2nd Edition). Weismann A (Ed.). Clarendon Press, Oxford, UK, 21-22 (1891).

3 Hayflick L, Moorhead PH. The serial cultivation of human diploid cell strains. Exp. Cell Res. 25, 585-621 (1961).

4 Haff RF, Swim HE. Serial propagation of 3 strains of rabbit fibroblasts; their susceptibility to infection with vaccinia virus. Proc. Soc. Exp. Biol. Med. 93(2), 200-204 (1956).

5 Goldstein S. Lifespan of cultured cells in progeria. Lancet 1(7591), 424 (1969).

6 Salk D, Au K, Hoehn H, Martin GM. Effects of radicalscavenging enzymes and reduced oxygen exposure on growth and chromosome abnormalities of Werner syndrome cultured skin fibroblasts. Hum. Genet. 57(3), 269-275 (1981).

7 West MD, Pereira-Smith OM, Smith JR. Replicative senescence of human skin fibroblasts correlates with a loss of regulation and overexpression of collagenase activity. Exp. Cell Res. 184(1), 138-147 (1989).

8 West MD, Shay JW, Wright WE, Linskens MH. Altered expression of plasminogen activator and plasminogen activator inhibitor during cellular senescence. Exp. Gerontol. 31(1-2), 175-193 (1996).

9 West MD. The cellular and molecular biology of skin aging. Arch. Dermatol. 130(1), 87-95 (1994).

10 Mclaren A. Embryology. The quest for immortality. Nature 359(6395), 482-483 (1992).

11 Mclaren A. Mammalian germ cells: birth, sex, and immortality. Cell Struct. Funct. 26(3), 119-122 (2001).

12 Van Der Kooi CJ, Schwander T. Parthenogenesis: birth of a new lineage or reproductive accident? Curr. Biol. 25(15), R659-R661 (2015).

13 Avise JC. Evolutionary perspectives on clonal reproduction in vertebrate animals. Proc. Natl Acad. Sci. USA 112(29), 8867-8873 (2015).

14 Olovnikov AM. A theory of marginotomy. The incomplete copying of template margin in enzymic synthesis of polynucleotides and biological significance of the phenomenon. J. Theor. Biol. 41(1), 181-190 (1973).

15 Olovnikov AM. [Principle of marginotomy in template synthesis of polynucleotides]. Dokl Akad Nauk SSSR 201(6), 1496-1499 (1971).

16 Dell'Orco RT, Mertens JG, Kruse PF Jr. Doubling potential, calendar time, and senescence of human diploid cells in culture. Exp. Cell Res. 77(1), 356-360 (1973).

17 Cooke HJ, Smith BA. Variability at the telomeres of the human X/Y pseudoautosomal region. Cold Spring Harb. Symp. Quant. Biol. 51(Pt 1), 213-219 (1986).

18 Vaziri H, Dragowska W, Allsopp RC, Thomas TE, Harley CB, Lansdorp PM. Evidence for a mitotic clock in human hematopoietic stem cells: loss of telomeric DNA with age. Proc. Natl Acad. Sci. USA 91(21), 9857-9860 (1994).
19 Harley CB, Futcher AB, Greider CW. Telomeres shorten during ageing of human fibroblasts. Nature 345(6274), 458-460 (1990).

20 Counter CM, Avilion AA, Lefeuvre CE et al. Telomere shortening associated with chromosome instability is arrested in immortal cells which express telomerase activity. EMBO J. 11(5), 1921-1929 (1992).

21 Harley CB, Kim NW, Prowse KR et al. Telomerase, cell immortality, and cancer. Cold Spring Harb. Symp. Quant. Biol. 59, 307-315 (1994).

22 Kim NW, Piatyszek MA, Prowse KR et al. Specific association of human telomerase activity with immortal cells and cancer. Science 266(5193), 2011-2015 (1994).

23 Ungewitter E, Scrable H. Antagonistic pleiotropy and p53. Mech. Ageing Dev. 130(1-2), 10-17 (2009).

24 Bodnar AG, Ouellette M, Frolkis M et al. Extension of lifespan by introduction of telomerase into normal human cells. Science 279(5349), 349-352 (1998).

25 Bai Y, Murnane JP. Telomere instability in a human tumor cell line expressing a dominant-negative WRN protein. Hum. Genet. 113(4), 337-347 (2003).

26 Allsopp RC, Vaziri H, Patterson C et al. Telomere length predicts replicative capacity of human fibroblasts. Proc. Natl Acad. Sci. USA 89(21), 10114-10118 (1992).

27 Kudlow BA, Stanfel MN, Burtner CR, Johnston ED, Kennedy BK. Suppression of proliferative defects associated with processing-defective lamin A mutants by hTERT or inactivation of p53. Mol. Biol. Cell 19(12), 5238-5248 (2008).

28 Du X, Shen J, Kugan N et al. Telomere shortening exposes functions for the mouse Werner and Bloom syndrome genes. Mol. Cell Biol. 24(19), 8437-8446 (2004).

29 Tomas-Loba A, Flores I, Fernandez-Marcos PJ et al. Telomerase reverse transcriptase delays aging in cancerresistant mice. Cell 135(4), 609-622 (2008).

30 Suram A, Herbig U. The replicometer is broken: telomeres activate cellular senescence in response to genotoxic stresses. Aging Cell 13(5), 780-786 (2014).

31 Hayflick L. Human cells and aging. Sci. Am. 218(3), 32-37 (1968).

32 Cristofalo VJ, Allen RG, Pignolo RJ, Martin BG, Beck JC. Relationship between donor age and the replicative lifespan of human cells in culture: a reevaluation. Proc. Natl Acad. Sci. USA 95(18), 10614-10619 (1998).

33 Yin D. Studies on age pigments evolving into a new theory of biological aging. Gerontology 41(Suppl. 2), 159-172 (1995).

34 Cristofalo VJ, Kabakjian J. Lysosomal enzymes and aging in vitro: subcellular enzyme distribution and effect of hydrocortisone on cell life-span. Mech. Ageing Dev. 4(1), 19-28 (1975).

35 Itahana K, Campisi J, Dimri GP. Methods to detect biomarkers of cellular senescence: the senescence-associated beta-galactosidase assay. Methods Mol. Biol. 371, 21-31 (2007).

36 Sharpless NE, Sherr CJ. Forging a signature of in vivo senescence. Nat. Rev. Cancer 15(7), 397-408 (2015). 
37 Rai R, Chang S. Probing the telomere damage response. Methods Mol. Biol. 735, 145-150 (2011).

38 Nakamura AJ, Chiang YJ, Hathcock KS et al. Both telomeric and non-telomeric DNA damage are determinants of mammalian cellular senescence. Epigenetics Chromatin 1(1), 6 (2008).

39 Saha B, Zitnik G, Johnson $S$ et al. DNA damage accumulation and TRF2 degradation in atypical Werner syndrome fibroblasts with $L M N A$ mutations. Front. Genet. 5(4), 129 (2013).

40 Gonzalo S, Kreienkamp R. DNA repair defects and genome instability in Hutchinson-Gilford Progeria Syndrome. Curr. Opin. Cell Biol. 34, 75-83 (2015).

41 Lopez-Otin C, Blasco MA, Partridge L, Serrano M, Kroemer G. The hallmarks of aging. Cell 153(6), 1194-1217 (2013).

42 Thomson JA, Itskovitz-Eldor J, Shapiro SS et al. Embryonic stem cell lines derived from human blastocysts. Science 282(5391), 1145-1147 (1998).

43 Vaziri H, Chapman KB, Guigova A et al. Spontaneous reversal of the developmental aging of normal human cells following transcriptional reprogramming. Regen. Med. 5(3), 345-363 (2010).

44 De Coppi P, Bartsch G Jr, Siddiqui MM et al. Isolation of amniotic stem cell lines with potential for therapy. Nat. Biotechnol. 25(1), 100-106 (2007).

45 Kucia M, Reca R, Campbell FR et al. A population of very small embryonic-like (VSEL) CXCR $4^{+}$SSEA- $1^{+} \mathrm{Oct}-4^{+}$ stem cells identified in adult bone marrow. Leukemia 20(5), 857-869 (2006).

46 Miyanishi M, Mori Y, Seita J et al. Do pluripotent stem cells exist in adult mice as very small embryonic stem cells? Stem Cell Reports 1(2), 198-208 (2013).

47 Wilmut I, Schnieke AE, McWhir J, Kind AJ, Campbell KH. Viable offspring derived from fetal and adult mammalian cells. Nature 385(6619), 810-813 (1997).

48 Lanza RP, Cibelli JB, West MD. Prospects for the use of nuclear transfer in human transplantation. Nat. Biotechnol. 17(12), 1171-1174 (1999).

49 Shiels PG, Kind AJ, Campbell KH et al. Analysis of telomere lengths in cloned sheep. Nature 399(6734), 316-317 (1999).

50 Lanza RP, Cibelli JB, Blackwell C et al. Extension of cell lifespan and telomere length in animals cloned from senescent somatic cells. Science 288(5466), 665-669 (2000).

51 Miyashita N, Shiga K, Yonai M et al. Remarkable differences in telomere lengths among cloned cattle derived from different cell types. Biol. Reprod. 66(6), 1649-1655 (2002).

52 Takahashi K, Tanabe K, Ohnuki M et al. Induction of pluripotent stem cells from adult human fibroblasts by defined factors. Cell 131(5), 861-872 (2007).

53 Niedernhofer LJ, Glorioso JC, Robbins PD. Dedifferentiation rescues senescence of progeria cells but only while pluripotent. Stem Cell Res. Ther. 2(3), 28 (2011).

54 Shimamoto A, Kagawa H, Zensho K et al. Reprogramming suppresses premature senescence phenotypes of Werner syndrome cells and maintains chromosomal stability over long-term culture. PLoS ONE 9(11), e112900 (2014).
55 Funk WD, Labat I, Sampathkumar J et al. Evaluating the genomic and sequence integrity of human ES cell lines; comparison to normal genomes. Stem Cell Res. 8(2), 154-164 (2012).

56 Rong Z, Wang M, Hu Z et al. An effective approach to prevent immune rejection of human ESC-derived allografts. Cell Stem Cell 14(1), 121-130 (2014).

57 Riolobos L, Hirata RK, Turtle CJ et al. HLA engineering of human pluripotent stem cells. Mol. Ther. 21(6), 1232-1241 (2013).

58 Walmsley GG, Maan ZN, Wong VW et al. Scarless wound healing: chasing the holy grail. Plast. Reconstr. Surg. 135(3), 907-917 (2015).

59 Sousounis K, Baddour JA, Tsonis PA. Aging and regeneration in vertebrates. Curr. Top Dev. Biol. 108, 217-246 (2014).

60 Fink DW Jr. FDA regulation of stem cell-based products. Science 324(5935), 1662-1663 (2009).

61 West MD, Mason C. Mapping the human embryome: 1 to $10 \mathrm{e} 13$ and all the cells in between. Regen. Med. 2(4), 329-333 (2007).

62 West MD, Sargent RG, Long J et al. The ACTCellerate initiative: large-scale combinatorial cloning of novel human embryonic stem cell derivatives. Regen. Med. 3(3), 287-308 (2008).

63 Sternberg H, Kidd J, Murai JT et al. Seven diverse human embryonic stem cell-derived chondrogenic clonal embryonic progenitor cell lines display site-specific cell fates. Regen. Med. 8(2), 125-144 (2013).

64 Sternberg H, Jiang J, Sim P et al. Human embryonic stem cell-derived neural crest cells capable of expressing markers of osteochondral or meningeal-choroid plexus differentiation. Regen. Med. 9(1), 53-66 (2014).

65 Sternberg H, Murai JT, Erickson IE et al. A human embryonic stem cell-derived clonal progenitor cell line with chondrogenic potential and markers of craniofacial mesenchyme. Regen. Med. 7(4), 481-501 (2012).

66 Reddig PJ, Juliano RL. Clinging to life: cell to matrix adhesion and cell survival. Cancer Metastasis Rev. 24(3), 425-439 (2005).

67 Prestwich GD, Erickson IE, Zarembinski TI, West M, Tew WP. The translational imperative: making cell therapy simple and effective. Acta Biomater. 8(12), 4200-4207 (2012).

68 Herbig U, Ferreira M, Condel L, Carey D, Sedivy JM. Cellular senescence in aging primates. Science 311(5765), 1257 (2006)

69 Mogford JE, Liu WR, Reid R et al. Adenoviral human telomerase reverse transcriptase dramatically improves ischemic wound healing without detrimental immune response in an aged rabbit model. Hum. Gene Ther. 17(6), 651-660 (2006).

70 Lo DD, Zimmermann AS, Nauta A, Longaker MT, Lorenz HP. Scarless fetal skin wound healing update. Birth Defects Res. CEmbryo Today 96(3), 237-247 (2012).

71 Lawrence RC, Felson DT, Helmick CG et al. Estimates of the prevalence of arthritis and other rheumatic conditions 
in the United States. Part II. Arthritis Rheum. 58(1), 26-35 (2008).

72 Shane Anderson A, Loeser RF. Why is osteoarthritis an age-related disease? Best Pract. Res. Clin. Rheumatol. 24(1), 15-26 (2010).

73 Le Maitre CL, Freemont AJ, Hoyland JA. Accelerated cellular senescence in degenerate intervertebral discs: a possible role in the pathogenesis of intervertebral disc degeneration. Arthritis Res. Ther. 9(3), R45 (2007).

74 Jeong SW, Lee JS, Kim KW. In vitro lifespan and senescence mechanisms of human nucleus pulposus chondrocytes. Spine J. 14(3), 499-504 (2014)

75 Sternberg H, Kidd J, Murai JT et al. Seven diverse human embryonic stem cell-derived chondrogenic clonal embryonic progenitor cell lines display site-specific cell fates. Regen. Med. 8(2), 125-144 (2013).

76 Friedman DS, O'Colmain BJ, Munoz B et al. Prevalence of age-related macular degeneration in the United States. Arch Ophthalmol. 122(4), 564-572 (2004).

Dorey CK, Wu G, Ebenstein D, Garsd A, Weiter JJ. Cell loss in the aging retina. Relationship to lipofuscin accumulation and macular degeneration. Invest. Ophthalmol. Vis. Sci. 30(8), 1691-1699 (1989).

78 McLeod DS, Taomoto M, Otsuji T, Green WR, Sunness JS, Lutty GA. Quantifying changes in RPE and choroidal vasculature in eyes with age-related macular degeneration. Invest. Ophthalmol. Vis. Sci. 43(6), 1986-1993 (2002).

79 Dawson DW, Volpert OV, Gillis P et al. Pigment epitheliumderived factor: a potent inhibitor of angiogenesis. Science 285(5425), 245-248 (1999).

80 Matsunaga H, Handa JT, Aotaki-Keen A, Sherwood SW, West MD, Hjelmeland LM. Beta-galactosidase histochemistry and telomere loss in senescent retinal pigment epithelial cells. Invest. Ophthalmol. Vis. Sci. 40(1), 197-202 (1999).

81 Doggett DL, Rotenberg MO, Pignolo RJ, Phillips PD, Cristofalo VJ. Differential gene expression between young and senescent, quiescent WI-38 cells. Mech Ageing Dev. 65(2-3), 239-255 (1992).

82 Ferrington DA, Sinha D, Kaarniranta K. Defects in retinal pigment epithelial cell proteolysis and the pathology associated with age-related macular degeneration. Prog. Retin. Eye Res. 51, 69-89 (2015).

83 Da Cruz L, Chen FK, Ahmado A, Greenwood J, Coffey P. RPE transplantation and its role in retinal disease. Prog. Retin. Eye Res. 26(6), 598-635 (2007).

84 Klimanskaya I, Hipp J, Rezai KA, West M, Atala A, Lanza R. Derivation and comparative assessment of retinal pigment epithelium from human embryonic stem cells using transcriptomics. Cloning Stem Cells 6(3), 217-245 (2004).

85 Idelson M, Alper R, Obolensky A et al. Directed differentiation of human embryonic stem cells into functional retinal pigment epithelium cells. Cell Stem Cell 5(4), 396-408 (2009).

86 Lund RD, Wang S, Klimanskaya I et al. Human embryonic stem cell-derived cells rescue visual function in dystrophic RCS rats. Cloning Stem Cells 8(3), 189-199 (2006).
87 Garber K. RIKEN suspends first clinical trial involving induced pluripotent stem cells. Nat. Biotechnol. 33(9), 890-891 (2015).

88 Xian B, Huang B. The immune response of stem cells in subretinal transplantation. Stem Cell Res. Ther. 6(1), 161 (2015).

89 Lopez-Leon M, Reggiani PC, Herenu CB, Goya RG. Regenerative medicine for the aging brain. Enliven. J. Stem Cell Res. Regen. Med. 1(1), 1-9 (2014).

90 Lindvall O. Clinical translation of stem cell transplantation in Parkinson's disease. J. Intern. Med. 279(1), 30-40 (2016).

91 Grealish S, Diguet E, Kirkeby A et al. Human ESC-derived dopamine neurons show similar preclinical efficacy and potency to fetal neurons when grafted in a rat model of Parkinson's disease. Cell Stem Cell 15(5), 653-665 (2014).

92 Dodson MV, Du M, Wang S et al. Adipose depots differ in cellularity, adipokines produced, gene expression, and cell systems. Adipocyte 3(4), 236-241 (2014).

93 Xiong ZM, Ladana C, Wu D, Cao K. An inhibitory role of progerin in the gene induction network of adipocyte differentiation from iPS cells. Aging (Albany NY) 5(4), 288-303 (2013).

94 Mori S, Murano S, Yokote K et al. Enhanced intra-abdominal visceral fat accumulation in patients with Werner's syndrome. Int. J. Obes. Relat. Metab. Disord. 25(2), 292-295 (2001).

95 Yoneshiro T, Aita S, Matsushita M et al. Age-related decrease in cold-activated brown adipose tissue and accumulation of body fat in healthy humans. Obesity (Silver Spring) 19(9), 1755-1760 (2011).

96 Mattson MP. Perspective: does brown fat protect against diseases of aging? Ageing Res. Rev. 9(1), 69-76 (2010).

97 Sternberg H, Janus J, West MD. Defining cell-matrix combination products in the era of pluripotency. Biomatter 3(1), e24496 (2013).

98 Cawthon RM, Smith KR, O’Brien E, Sivatchenko A, Kerber RA. Association between telomere length in blood and mortality in people aged 60 years or older. Lancet 361(9355), 393-395 (2003).

99 Lin J, Epel E, Blackburn E. Telomeres and lifestyle factors: roles in cellular aging. Mutat. Res. 730(1-2), 85-89 (2012).

100 Canela A, Vera E, Klatt P, Blasco MA. High-throughput telomere length quantification by FISH and its application to human population studies. Proc. Natl Acad. Sci. USA 104(13), 5300-5305 (2007).

101 Bendix L, Horn PB, Jensen UB, Rubelj I, Kolvraa S. The load of short telomeres, estimated by a new method, Universal STELA, correlates with number of senescent cells. Aging Cell 9(3), 383-397 (2010).

102 Vaziri H, Schachter F, Uchida I et al. Loss of telomeric DNA during aging of normal and trisomy 21 human lymphocytes. Am. J. Hum. Genet. 52(4), 661-667 (1993).

103 Effros RB, Allsopp R, Chiu CP et al. Shortened telomeres in the expanded CD28-CD $8{ }^{+}$cell subset in HIV disease implicate replicative senescence in HIV pathogenesis. AIDS 10(8), F17-F22 (1996).

104 Chirch LM, Hasham M, Kuchel GA. HIV and aging: a clinical journey from Koch's postulate to the chronic 
disease model and the contribution of geriatric syndromes. Curr. Opin. HIV AIDS 9(4), 405-411 (2014).

105 Gadalla SM, Wang T, Haagenson M et al. Association between donor leukocyte telomere length and survival after unrelated allogeneic hematopoietic cell transplantation for severe aplastic anemia. JAMA 313(6), 594-602 (2015).

106 Goligorsky MS. Endothelial progenitor cells: from senescence to rejuvenation. Semin. Nephrol. 34(4), 365-373 (2014).

107 Kushner EJ, Van Guilder GP, Maceneaney OJ, Cech JN, Stauffer BL, Desouza CA. Aging and endothelial progenitor cell telomere length in healthy men. Clin. Chem. Lab. Med. 47(1), 47-50 (2009).

108 Thom T, Haase N, Rosamond W et al. Heart disease and stroke statistics - 2006 update: a report from the American
Heart Association Statistics Committee and Stroke Statistics Subcommittee. Circulation 113(6), e85-e151 (2006).

109 Lanza R, Shieh JH, Wettstein PJ et al. Long-term bovine hematopoietic engraftment with clone-derived stem cells. Cloning Stem Cells 7(2), 95-106 (2005).

110 Weiss CO. Frailty and chronic diseases in older adults. Clin. Geriatr. Med. 27(1), 39-52 (2011).

111 Hayflick L. Biological aging is no longer an unsolved problem. Ann. NY Acad. Sci. 1100, 1-13 (2007).

112 Centers for disease control and prevention. Chronic disease overview.

www.cdc.gov/chronicdisease/overview/ 